\title{
La prise en compte de la vie familiale des salariés par les entreprises Une comparaison européenne
}

Link to publication record in Manchester Research Explorer

\section{Citation for published version (APA):}

Letablier, M-T., Perraudin, C., Anxo, D., Fagan, C., \& Smith, M. (2008). La prise en compte de la vie familiale des salariés par les entreprises Une comparaison européenne. Recherches et Previsions, 92.

\section{Published in:}

Recherches et Previsions

\section{Citing this paper}

Please note that where the full-text provided on Manchester Research Explorer is the Author Accepted Manuscript or Proof version this may differ from the final Published version. If citing, it is advised that you check and use the publisher's definitive version.

\section{General rights}

Copyright and moral rights for the publications made accessible in the Research Explorer are retained by the authors and/or other copyright owners and it is a condition of accessing publications that users recognise and abide by the legal requirements associated with these rights.

\section{Takedown policy}

If you believe that this document breaches copyright please refer to the University of Manchester's Takedown Procedures [http://man.ac.uk/04Y6Bo] or contact uml.scholarlycommunications@manchester.ac.uk providing relevant details, so we can investigate your claim.

\section{OPEN ACCESS}




\section{La prise en compte de la vie familiale des} salariés par les entreprises : une comparaison européenne

Article · June 2008

DOI: $10.3406 /$ caf.2008.2374

CITATIONS

2

5 authors, including:

\section{Dominique Anxo}

Linnaeus University

109 PUBLICATIONS 604 CITATIONS

SEE PROFILE

\section{Colette Fagan}

The University of Manchester

126 PUBLICATIONS 1,820 CITATIONS

SEE PROFILE
READS

21

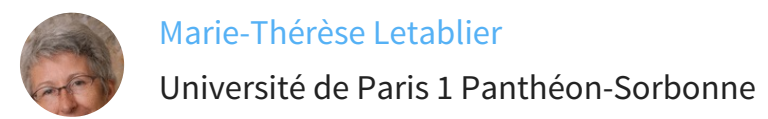

56 PUBLICATIONS 278 CITATIONS

SEE PROFILE

Mark Smith

Grenoble École de Management

57 PUBLICATIONS 719 CITATIONS

SEE PROFILE 


\title{
La prise en compte de la vie familiale des salariés par les entreprises : Une comparaison européenne
}

\author{
Marie-Thérèse Letablier $\quad$ Sociologue. Directrice de recherche au CNRS, Centre \\ d'économie de la Sorbonne/Matisse, université de Paris-1. \\ Corinne Perraudin \\ Centre d'études de l'emploi et Samos-Matisse-CES, \\ Université Paris-1. \\ Dominique Anxo \\ Centre for European labour Market Studies, Göteborg and \\ School of Management and Economics, Centre for Labour \\ Market Policy Research, Vaxjo University, Suède. \\ Colette Fagan \\ European Work and Employment Research Centre, \\ University of Manchester, Royaume-Uni. \\ Mark Smith \\ Professeur associé, École de management, chambre \\ de commerce et d'industrie de Grenoble.
}

L

organisation des temps de travail des salariés

au sein des entreprises peut constituer un véritable outil de gestion de la main-d'œuvre. En effet, I'organisation temporelle du travail peut répondre aux besoins de flexibilité des entreprises (aménagement des temps de travail selon les besoins organisationnels de l'entreprise, selon les variations de l'activité), ou bien être utilisée comme moyen de fidéliser des salariés, de renforcer leur satisfaction et de stimuler leur implication dans le travail en prenant en compte leurs obligations personnelles. Qu'en est-il réellement? Quelles sont les pratiques des entreprises en matière de conciliation? De nombreuses études ont analysé les pratiques individuelles des salariés - notamment lorsqu'ils ont des responsabilités parentales ou familiales - en soulignant la diversité des dispositifs institutionnels en Europe (Fagan, 2003), mais peu de recherches ont été consacrées aux pratiques des entreprises. Les recherches comparatives notamment se sont concentrées sur le rôle respectif de l'État, des familles et du marché dans l'offre de conciliation et ont quelque peu délaissé les entreprises dans l'analyse de l'articulation travail et vie familiale. Une des raisons est le manque d'enquêtes permettant de fournir des données comparables sur cette question.

L'enquête ESWT (Establishment Survey on Working-Time and Work-Life Balance) de la Fondation de Dublin est une des rares enquêtes européennes auprès des entreprises qui apporte un grand nombre d'informations qualitatives sur les pratiques de ces dernières en matière de temps de travail et de conditions de vie (encadré, p. 92). L'exploitation d'une partie des résultats de I'enquête permet de comparer les pratiques des entreprises en Europe en matière d'articulation entre vie professionnelle et vie familiale. Quatre

aspects sont plus particulièrement étudiés dans cet article : les arrangements temporels, la diffusion des congés parentaux, le recours au travail à temps partiel ainsi que les offres de services aux salariés.

\section{Les aménagements d'horaires : davantage proposés dans les secteurs des services}

La possibilité d'aménager ses horaires peut permettre aux salariés de mieux articuler vies familiale et professionnelle en adaptant, dans une certaine limite, les heures d'arrivée et de départ du travail. En outre, la possibilité de compenser des heures accumulées en réduisant d'autant la durée du travail certains autres jours ou certaines semaines peut offrir aux salariés une certaine souplesse dans l'organisation de leur temps de travail. Ces possibilités peuvent être prévues dans des accords collectifs ou bien relever de régulations législatives (loi sur les 35 heures en France). Elles peuvent aussi résulter d'arrangements individuels plus ou moins formalisés entre l'employeur et le salarié, mais la manière dont elles sont mises en place dépend largement des pratiques des entreprises et du dialogue social. L'enquête fournit des informations sur ces arrangements ou «possibilités $\mathrm{d}^{\prime}$ adaptation selon les besoins personnels des salariés ». En moyenne, sur les vingt et un pays de I'Union européenne (UE) concernés par I'enquête, $48 \%$ des employeurs de dix salariés et plus déclarent que de tels arrangements existent dans leur établissement (graphique 1, p. 93). La forme la moins flexible (compenser dans la même journée les horaires décalés) est la plus répandue (16\% d'établissements). Viennent ensuite les possibilités d'accumuler des heures, 


\section{L'enquête Establishment Survey on Working-Time and Work-Life Balance, 2004-2005}

L'enquête Establishment Survey on Working-Time and Work-Life Balance (ESWT) a été réalisée par I'institut TNS Infratest Sozialforschung (Munich, Allemagne) pour le compte de la Fondation européenne pour l'amélioration des conditions de vie et de travail (http://www.eurofound.europa.eu/areas/ worklifebalance/eswt.htm). Cette enquête a été menée dans les établissements de plus de dix salariés de vingt et un pays de l'Union européenne (UE), en 2004 pour les quinze pays qui formaient I'UE jusqu'au $1^{\text {er }}$ mai 2004, et en 2005 au sein de six pays parmi les dix entrants au $1^{\mathrm{er}}$ mai 2004 : Chypre, Hongrie, Lettonie, Pologne, République Tchèque et Slovénie. Il s'agit d'une enquête couplée employeurs et représentants du personnel. Au total, 21031 établissements de dix salariés et plus de tous les secteurs sauf I'agriculture (21 031 représentants de la direction et 5232 représentants du personnel) ont été interrogés, représentatifs de plus de 2,7 millions d'établissements.

Au-delà des renseignements sur les caractéristiques de l'établissement, l'enquête fournit des informations sur le temps partiel, les horaires atypiques, les arrangements de temps de travail flexibles, les heures supplémentaires, les congés parentaux, les préretraites [pour une présentation générale de l'enquête et les principaux résultats voir Riedmann, 2006 (1)]. Chaque thème a donné lieu à une étude spécifique : voir Anxo et al., 2007 a pour les congés parentaux (2) et 2007 b pour le temps partiel (3); Kümmerling et Lehndorff, 2007, pour les horaires atypiques et les heures supplémentaires (4); Leber et Wagner, 2007, pour les retraites anticipées ou progressives (5) ; Chung et al., 2007, pour la flexibilité au niveau des établissements reprenant les quatre thèmes précédents (6); Lehndorff et al., pour une étude plus spécifique du dialogue social sur le temps de travail et la conciliation) (7).

Cependant, la richesse de l'enquête, à la fois en termes de pays concernés et de thèmes traités, fait que certains points n'ont pas été renseignés. Notamment, le temps partiel correspond à une durée inférieure au temps de travail usuel; on ne peut ainsi distinguer un temps partiel court d'un temps partiel long. L'enquête ne fournit qu'une information générale sur la présence de divers groupes de salariés à temps partiel (mères avec enfants en bas âge, femmes sans enfant ou mères de grands enfants, pères, handicapés, personnes s'occupant de proches dépendants, étudiants, jeunes) et ne donne pas la proportion de chaque type de salarié à temps partiel. Les informations qualitatives, par exemple sur les raisons principales de l'introduction du temps partiel, des aménagements horaires, les possibilités de passage à temps partiel ou à temps complet, sont renseignées pour tous les salariés. Concernant les congés parentaux, seul le fait qu'au moins un salarié ait pris un tel congé sur les trois années précédant l'enquête est fourni, mais aucune information sur l'intensité n'est donnée. Enfin, ne portant que sur une année, l'enquête ne permet pas une approche dynamique des pratiques d'entreprises.

(1) Riedmann A., 2006, Working time and work-life balance in European companies, Establishment Survey of Working Time 2004-2005, European Foundation for the Improvement of Living and Working Conditions, Luxembourg: Office for Official Publications of the European Communities.

(2) Anxo D., Fagan C., Letablier M.-T., Perraudin C. et Smith M. , 2007 a, Parental leave in european companies. Establishment Survey of Working Time 2004-2005, European Foundation for the Improvement of Living and Working Conditions, Luxembourg Office for Official Publications of the European Communities. (3) Anxo D., Fagan C., Letablier M.-T., Perraudin C. et Smith M., 2007 b, Part-time work in european companies. Establishment Survey of Working Time 2004-2005, European Foundation for the Improvement of Living and Working Conditions, Luxembourg, Office for Official Publications of the European Communities. (4) Kümmerling A. et Lehndorff S., 2007, Extended and unusual working hours in european companies. Establishment Survey of Working Time 2004-2005, European Foundation for the Improvement of Living and Working Conditions, Luxembourg Office for Official Publications of the European Communities. (5) Leber U. et Wagner A., 2007, Early and phased retirement in european companies. Establishment Survey of Working Time 2004-2005, European Foundation for the Improvement of Living and Working Conditions, Luxembourg, Office for Official Publications of the European Communities.

(6) Chung H., Kerkhofs M. et Ester P., 2007, Working time flexibility in European companies. Establishment survey of working time 2004-2005, European Foundation for the Improvement of Living and Working Conditions, Luxembourg, Office for Official Publications of the European Communities..

(7) Lehndorff S., Kümmerling A. et Ramioul M., 2008, Social Dialogue. Establishment Survey of Working Time 2004-2005, European Foundation for the Improvement of Living and Working Conditions, Luxembourg, Office for Official Publications of the European Communities (à paraître). en les récupérant sur des plages longues pour $13 \%$ des établissements (compte épargne temps ou temps de travail annualisé), sur des journées entières $(12 \%)$, et en travaillant moins certains autres jours (7\%).

La proportion d'établissements déclarant offrir la possibilité d'arrangements horaires, quelle que soit la modalité, est plus importante dans les secteurs des services (50\%) que dans l'industrie (43\%). Ce type d'arrangement est le moins pratiqué dans la construction ( $36 \%$ ) alors que plus de six établissements sur dix offrent ces possibilités dans les activités immobilières, les services collectifs, les services sociaux et aux personnes, les banques et les administrations publiques.

\section{Des disparités importantes entre le nord et le sud de I'Europe}

Des variations nationales opposent les pays du nord et ceux du sud de l'Europe : moins d'un tiers des établissements à Chypre, au Portugal et en Grèce proposent de telles possibilités, alors qu'ils sont plus des deux tiers en Suède, en Lettonie ou en Finlande. Dans les autres pays, la proportion d'établissements offrant ces possibilités est comprise entre $40 \%$ et $55 \%$. Le degré de flexibilité de ces arrangements, à savoir les possibilités ou non de compenser des horaires plus longs, varie également entre les pays. Les formes les plus flexibles d'arrangements sont plus répandues dans les établissements des pays nordiques, ainsi qu'en Allemagne, en Autriche ou en République tchèque. 
En revanche, les possibilités d'adapter les horaires journaliers sont plus répandues dans les pays où peu d'établissements offrent en général de tels arrangements horaires, comme dans les pays du sud de I'Europe. Toutefois, cette forme moins flexible est particulièrement diffusée au Royaume-Uni et en Irlande.

\section{Des arrangements qui ne touchent pas l'ensemble des salariés}

Bien que l'aménagement des horaires de travail soit une pratique courante, les établissements qui permettent de telles possibilités ne l'accordent pas nécessairement à tous les salariés. Dans $40 \%$ de ces établissements, ces possibilités sont offertes à la totalité des employés, alors que dans $20 \%$ les choix ne sont donnés qu'à moins de $20 \%$ des salariés. En Suède ou en Finlande, ces possibilités sont offertes dans un grand nombre d'établissements et accessibles à un grand nombre de salariés: $60 \%$ des établissements donnent le choix à tous les salariés. En revanche, bien que des proportions élevées d'établissements offrent de telles possibilités en Lettonie, en Pologne, en République tchèque, ainsi qu'au Royaume-Uni et en Irlande, les arrangements concernent plus rarement I'ensemble des salariés (Riedmann, 2006, figure 4 page 7). Ces résultats tendent à nuancer l'introduction de tels arrangements dans le but de favoriser la conciliation de l'ensemble des salariés. L'aménagement des horaires pourrait être un outil de flexibilité utilisé selon les besoins des entreprises pour certaines catégories de salariés sur qui reposeraient plus particulièrement les ajustements nécessaires à l'entreprise.

\section{Dirigeants et représentants des salariés divergent sur les raisons du recours}

Les dirigeants d'entreprises ont été interrogés sur les raisons de l'introduction de tels arrangements flexibles (tableau 1, p.94). Les dirigeants pouvaient donner plusieurs raisons : permettre aux salariés de mieux articuler travail et vie familiale ou personnelle est la raison la plus fréquemment citée (68\% des établissements) ; rendre les heures de travail plus adaptées aux variations de l'activité est une réponse donnée par $47 \%$ des établissements ; réduire les heures supplémentaires payées (14\%) ou les problèmes de transports ( $22 \%)$. Si on isole les réponses qui concernent uniquement les souhaits des salariés de mieux concilier travail et famille de celles relatives uniquement aux besoins

Graphique 1 - Les différentes formes d'aménagement flexible des horaires (en \% d'établissements)

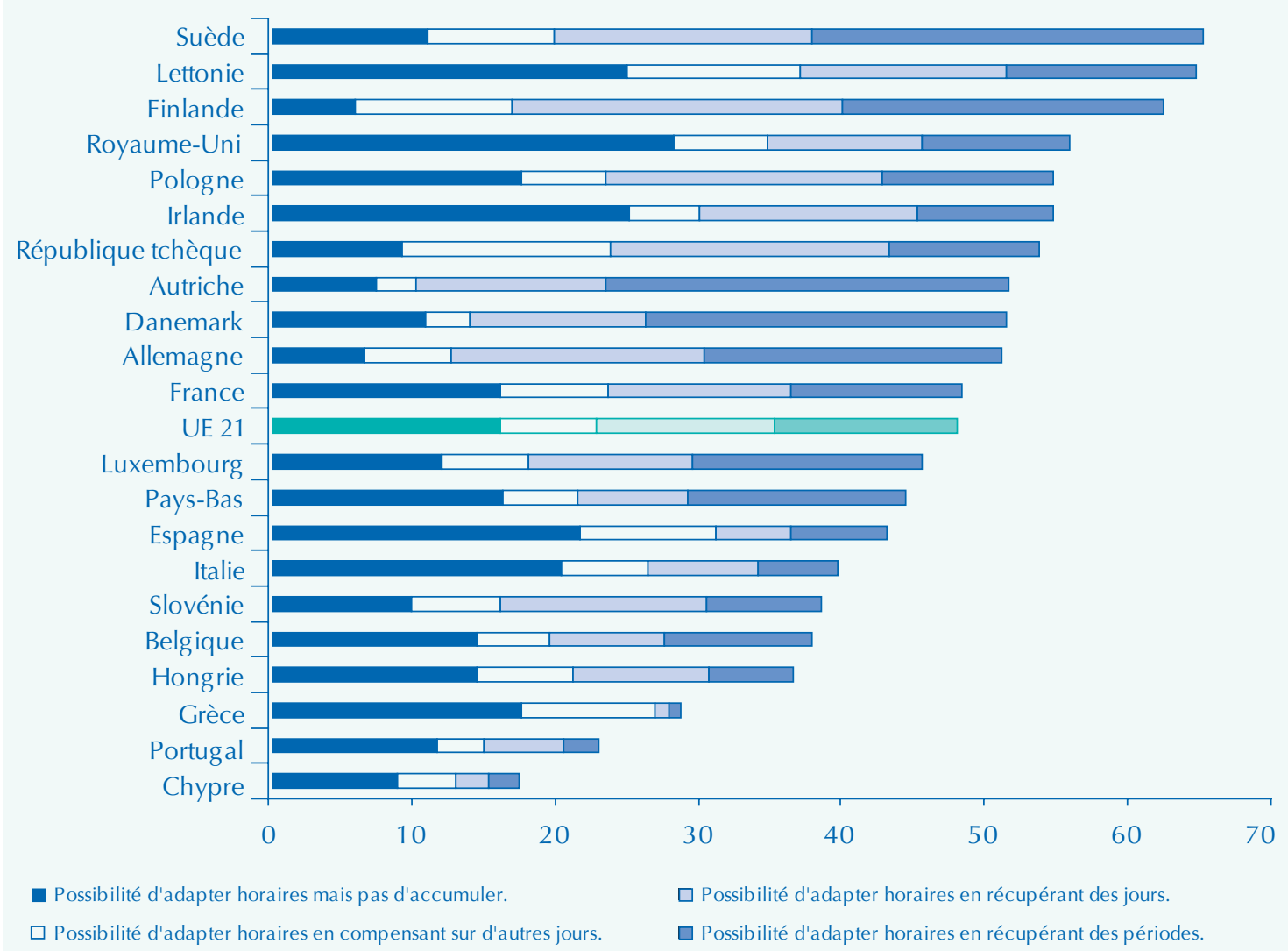

Source : enquête ESWT 2004-2005, Fondation européenne pour l'amélioration des conditions de vie et de travail.

Champ : établissements de dix salariés et plus de tous les secteurs sauf agriculture (représentants de la direction).

Lecture du graphique : 48 \% des établissements des vingt et un pays de l'Union européenne proposent des possibilités d'aménagement des horaires : $16 \%$ offrent cette possibilité mais sans pouvoir compenser ensuite, $7 \%$ en pouvant compenser sur d'autres jours, $12 \%$ en pouvant récupérer des jours entiers, et $13 \%$ en pouvant récupérer des périodes plus longues. 
Tableau 1 - Les raisons de l'introduction d'horaires flexibles (en \% d'établissements)

\begin{tabular}{l|c|c|c} 
& $\begin{array}{c}\text { Selon les } \\
\text { représentants } \\
\text { de la direction }\end{array}$ & $\begin{array}{c}\text { Selon les } \\
\text { représentants } \\
\text { de la direction } \\
\text { quand il y a un RP* }\end{array}$ & $\begin{array}{c}\text { Selon les } \\
\text { représentants } \\
\text { du personnel } \\
\text { quand il y a un RP* }\end{array}$ \\
\cline { 2 - 3 } Les raisons de l'introduction d'horaires flexibles & 68,2 & 73,2 & 71,8 \\
Rendre les horaires adaptés aux variations de l'activité & 46,9 & 55,4 & 57,6 \\
Gérer les problèmes de transport & 22,2 & 24,3 & 29,8 \\
Réduire les heures supplémentaires payées & 13,9 & 18,4 & 22,2 \\
Autres raisons & 9,4 & 10,3 & 18,2 \\
Ne sait pas & 3,2 & 2,1 & 2,8 \\
Les raisons (exclusif) & 29,5 & 24,2 & 19,7 \\
Seulement permettre aux salariés de mieux articuler travail et famille & 21,7 & 19,1 & 20,5 \\
Seulement répondre aux besoins de l'entreprise & 38,7 & 49,0 & 52,1 \\
Combinaison des deux & 10,1 & 7,8 & 7,7 \\
Autres ou ne sait pas &
\end{tabular}

Source : enquête ESWT 2004-2005, Fondation européenne pour l'amélioration des conditions de vie et de travail.

Champ : établissements de dix salariés et plus de tous les secteurs sauf agriculture (représentants de la direction et représentants du personnel). * RP : représentants du personnel.

Lecture du tableau : les horaires flexibles ont été introduits pour réduire les heures supplémentaires payées pour 13,9\% des employeurs. Cette raison est fournie par $18,4 \%$ des employeurs, quand on se restreint aux établissements dans lesquels il y a un représentant du personnel (RP) et par $22,2 \%$ des représentants du personnel dans ce même champ.

organisationnels des entreprises, alors $29 \%$ des établissements ne citent que l'objectif de mieux articuler travail et famille et $22 \%$ des raisons relevant des besoins d'organisation de l'entreprise. Pour $39 \%$ des établissements, les deux raisons sont données à la fois, répondre aux demandes des salariés et satisfaire les besoins de l'entreprise.

Si on considère les réponses des représentants des salariés, la hiérarchie des raisons s'efface : ils sont environ $20 \%$ à répondre uniquement I'une ou uniquement l'autre raison, et $52 \%$ à citer les deux types de raisons à la fois. Ce résultat tend à nuancer le fait que ces arrangements d'horaires seraient forcément bénéfiques pour les salariés en leur permettant de mieux concilier travail et vie hors travail. Pour les représentants des salariés, l'aménagement des horaires de travail peut également répondre aux besoins organisationnels de l'entreprise en rendant les heures travaillées plus adaptées aux variations de l'activité, ainsi qu'en réduisant le coût des heures supplémentaires. L'aménagement des horaires peut constituer un outil de flexibilité pour les entreprises. C'est particulièrement le cas dans les pays de l'Est, mais également en Autriche et en Allemagne, contrairement à la Suède, au Danemark, à la Lettonie où les employeurs invoquent plus souvent des raisons liées aux besoins de conciliation des salariés. On note également que les établissements offrent peu d'arrangements flexibles dans les pays du sud de l'Europe, mais lorsqu'ils le font, c'est plutôt pour répondre aux besoins des salariés (graphique 2).

\section{L'usage des congés parentaux : de fortes variations entre les pays}

Bien que I'articulation entre travail et vie hors travail ne se limite pas aux responsabilités familiales, pour une majorité de salariés, les responsabilités familiales, notamment la garde des enfants, sont au cœur de la problématique de la conciliation. Les modalités d'usage du congé parental sont alors centrales dans ce domaine. En dépit de la directive européenne de 1998 qui accorde à tous les salariés en Europe, homme ou femme, un droit à prendre un congé parental à la suite de la naissance ou de l'adoption d'un enfant, il existe de fortes variations entre les pays quant aux modalités d'usage du congé parental, liées notamment à sa durée et à sa rémunération (Anxo et al., 2007 a pour une présentation des législations nationales en matière de congés parentaux). En moyenne sur les vingt et un pays de l'UE étudiés, $51 \%$ des établissements de dix salariés et plus ont eu au moins un salarié en congé parental au cours des trois années précédant l'enquête. La présence d'au moins un salarié en congé parental dans l'établissement augmente bien entendu avec la taille de l'établissement, dépassant les $90 \%$ d'établissements quand la taille excède deux cents salariés.

Cependant, il est intéressant de noter que dans certains pays (Chypre, Italie, Portugal, Espagne ou Royaume-Uni), la proportion d'établissements de grande taille ayant eu au moins un salarié en congé parental reste relativement faible (moins de 


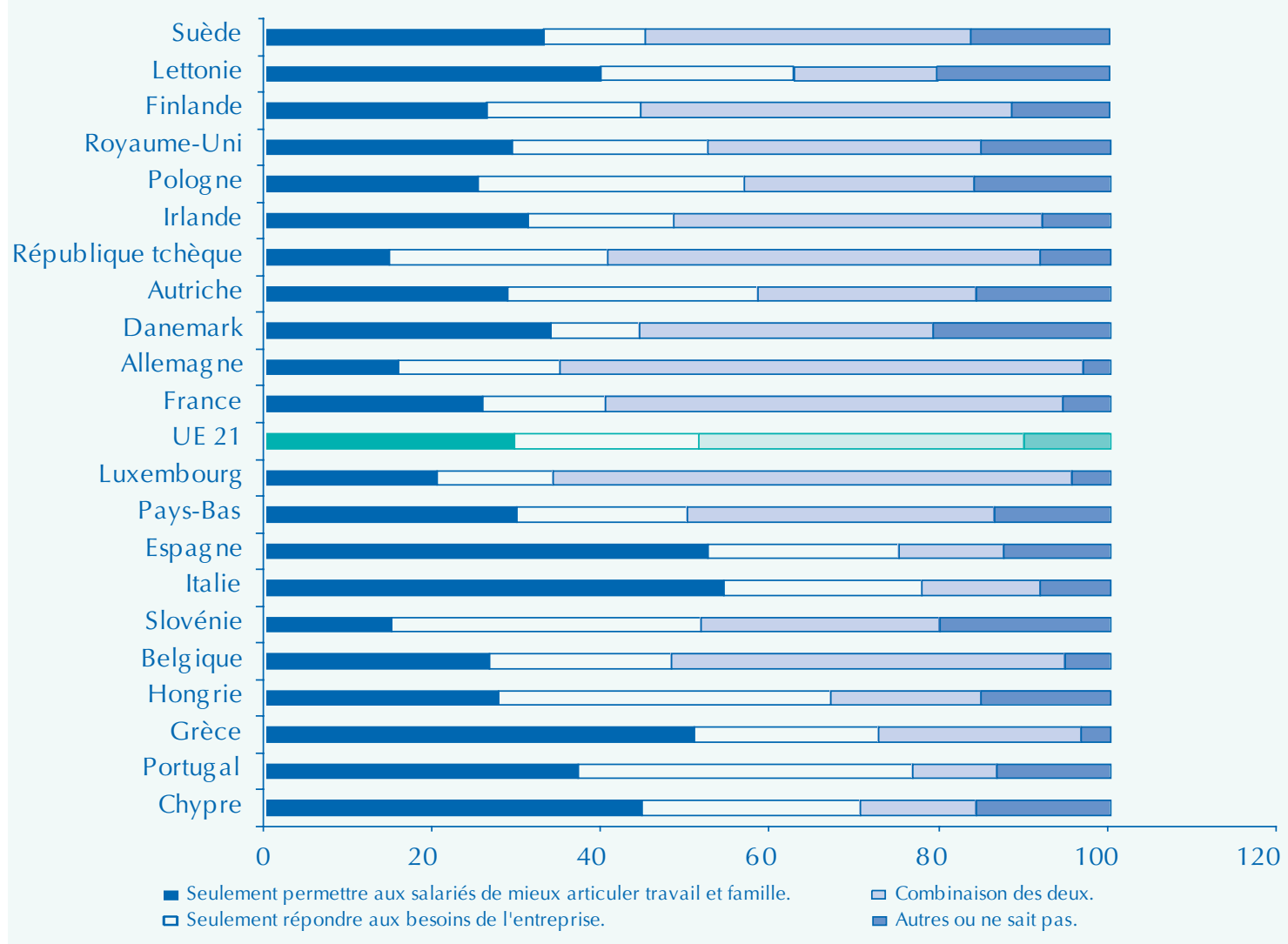

Source : enquête ESWT 2004-2005, Fondation européenne pour l'amélioration des conditions de vie et de travail.

Champ : établissements de dix salariés et plus de tous les secteurs sauf agriculture (représentants de la direction).

Les pays sont classés par usage de congés parentaux décroissant dans les établissements.

Lecture du graphique : En Suède, pour $12 \%$ des établissements, les raisons de l'introduction d'horaires flexibles fournies par les employeurs relèvent uniquement de motifs liés aux besoins de l'entreprise (réduire les heures supplémentaires payées, rendre les horaires adaptés aux variations de l'activité, ou gérer les problèmes de transport). Elles visent uniquement à permettre aux salariés de mieux articuler travail et famille pour $33 \%$ des établissements. Dans $39 \%$ des cas, les employeurs ont fourni les deux types de raison..

$80 \%)$, soulignant de fait que l'usage du congé parental reste plus limité dans ces pays. En revanche, on observe davantage d'établissements (de toutes tailles) concernés par des salariés en congés parentaux dans les pays nordiques (notamment en Suède et en Finlande) ou d'Europe de l'Ouest que dans les pays d'Europe du Sud ou de I'Est, à I'exception de la Slovénie (graphique 3, p. 96).

\section{Un usage fortement féminisé}

L'usage des congés parentaux reste principalement le fait de la mère. La présence de congés parentaux dans l'établissement augmente avec le taux de féminisation de la main-d'œuvre, et les secteurs les plus féminisés sont également ceux où l'on rencontre les proportions plus élevées d'établissements ayant eu des salariés en congés parentaux. Les établissements ayant octroyé des congés parentaux se trouvent davantage dans les services (55\%) que dans l'industrie (43\%), dans le secteur public (58\%) que dans le secteur privé $(49 \%)$. Certains pays cherchent à encourager les hommes à prendre des congés parentaux, en réservant par exemple une partie du congé aux pères. C'était le cas en 2005 en Suède, en Belgique, au Luxembourg ou en Slovénie. Dans d'autres pays, comme la Finlande, I'Italie ou l'Autriche, le congé parental est prolongé si le père en prend une partie. En moyenne, $30 \%$ des établissements ayant octroyé des congés parentaux indiquent qu'il y avait des hommes parmi les bénéficiaires de ces congés, mais avec de fortes variations entre les pays : de moins de $2 \%$ à Chypre ou en République tchèque à $59 \%$ en Finlande, $66 \%$ en Slovénie ou $69 \%$ en Suède, pays souvent cité comme exemple de participation des pères aux congés parentaux.

\section{Des difficultés de gestion des congés parentaux} Interrogés sur les difficultés qu'ils rencontrent dans la gestion des congés parentaux, les employeurs donnent des réponses nuancées selon les pays, reflétant les différences des contextes institutionnels, économiques et sociaux. Toutefois, la proportion d'établissements mentionnant des problèmes liés à la présence de salariés en congé parental reste relativement faible $(11 \%$ d'établissements). Le problème le plus fréquemment évoqué concerne les difficultés à remplacer les salariés absents ( $57 \%$ des établissements qui déclarent 
Graphique 3 - Présence de salariés en congés parentaux durant les trois années précédant l'enquête dans l'établissement, en fonction de la taille de l'établissement (en \% d'établissements)

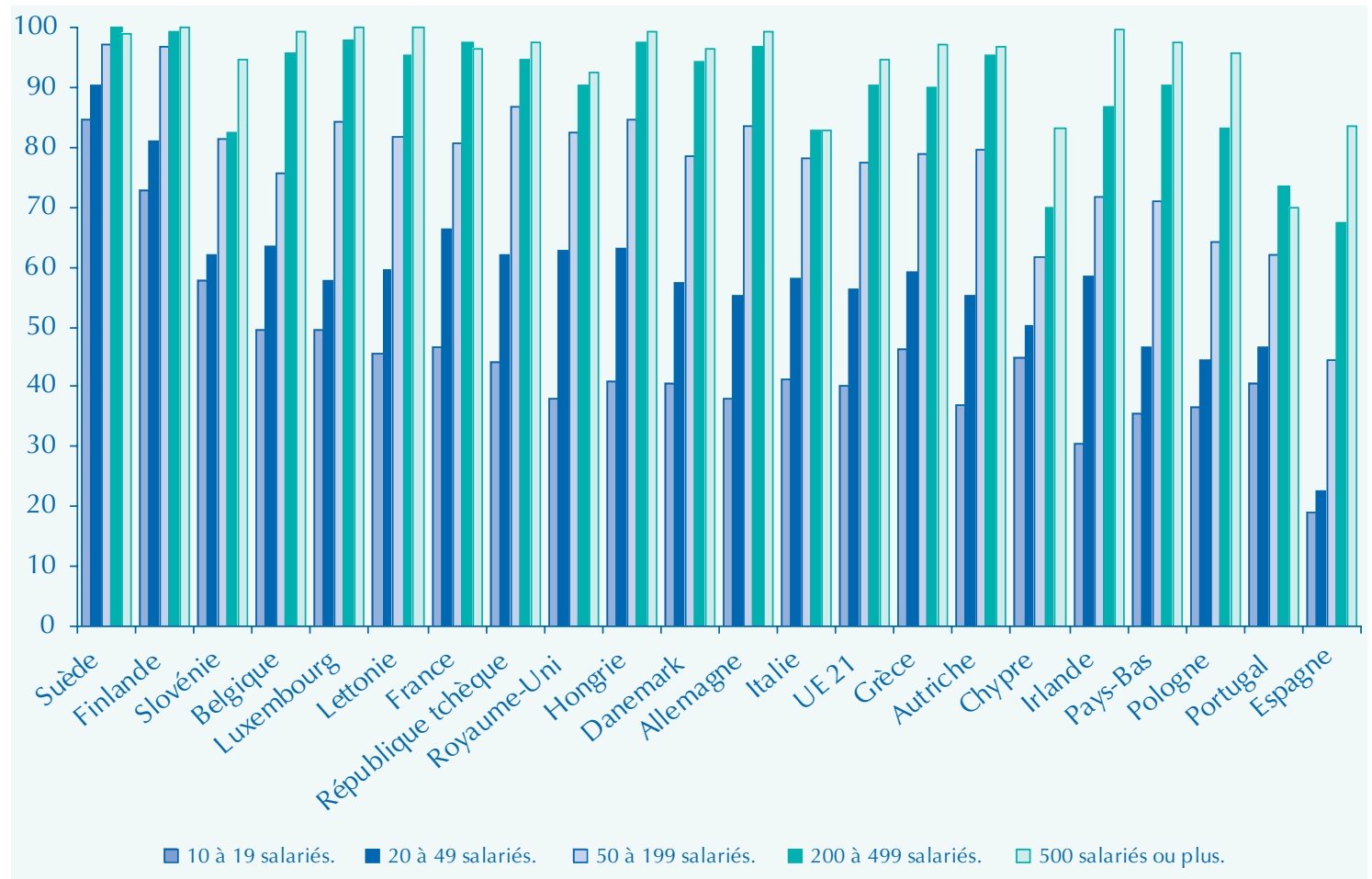

Source : enquête ESWT 2004-2005, Fondation européenne pour l'amélioration des conditions de vie et de travail. Champ : établissements de dix salariés et plus de tous les secteurs sauf agriculture (représentants de la direction). Les pays sont classés par usage de congés parentaux décroissant dans les établissements.

Lecture du graphique : En Suède, $84 \%$ des établissements de 10 à 19 salariés ont eu au moins un salarié en congé parental durant les trois années précédant l'enquête. La proportion est de $90 \%$ pour les établissements de 20 à 49 salariés, $97 \%$ pour ceux de 50 à 199 salariés, $100 \%$ pour ceux de 200 à 499 salariés, et $99 \%$ pour ceux de plus de 500 salariés.

rencontrer des problèmes), suivi par le manque de continuité au travail (52\%). En outre, $44 \%$ citent I'incertitude quant au retour du salarié, $24 \%$ le fait que trop de salariés soient absents, et $22 \%$ des difficultés à réintégrer les salariés au retour du congé parental. Au total, $37 \%$ des établissements énoncent trois problèmes ou plus à gérer. Globalement, la proportion d'établissements répondant qu'il y a des problèmes est plus faible dans les pays où les congés parentaux sont peu développés (sauf aux Pays-Bas). Pour les pays ayant des proportions plus importantes d'établissements avec des congés parentaux, des configurations diverses apparaissent quant aux problèmes qu'ils entraînent (graphique 4). Dans les pays de l'Est, les congés parentaux sont associés à moins de problèmes déclarés par les établissements, alors qu'au Luxembourg, en France, en Belgique ou en Allemagne, les établissements déclarent plus souvent rencontrer plusieurs problèmes à la fois. En Suède ou au Danemark, c'est plutôt un seul type de problème qui est cité (le plus souvent la difficulté à trouver du personnel pour remplacer les personnes absentes). Ces résultats soulignent le fait que les entreprises n'ont pas encore mis en place les moyens ou les modes d'organisation qui permettraient une diffusion plus grande de ces mesures.

\section{La majorité des mères reprennent leur emploi après un congé parental}

Dans un grand nombre d'établissements, la majorité des mères reprennent leur travail après leur congé parental dans les mêmes conditions qu'avant. $C^{\prime}$ est le cas dans $44 \%$ des établissements, alors que dans $34 \%$ des établissements, la majorité des femmes demandent un travail à temps partiel après leur congé parental, et seulement $10 \%$ indiquent que la majorité des femmes ne reprennent pas leur travail ou ne reviennent pas dans I'entreprise. Ces situations varient selon les pays. Reprendre le travail en demandant une réduction d'horaires est particulièrement répandu aux Pays-Bas, en Autriche, en Allemagne, en Suède et au Royaume-Uni, pays où I'incidence du travail à temps partiel est grande. Dans les autres pays, la plupart des mères retravaillent dans les mêmes conditions : c'est particulièrement le cas au Danemark, en Finlande, et dans la plupart des pays de I'Europe de I'Est et du Sud. II est plus fréquent que la majorité des mères ne retournent pas travailler (au moins dans la même entreprise) dans les pays de l'Est, ainsi qu'en Autriche et en Allemagne (Anxo et al., 2007 a). En moyenne, $22 \%$ des établissements offrent des programmes de formation aux salariés reprenant le travail après une longue période de congé pour motifs personnels. 
Cette proportion augmente avec la taille de I'entreprise pour atteindre $40 \%$ des très grands établissements (500 salariés et plus). Cette possibilité varie selon les pays : environ un établissement sur deux en Pologne et en Suède, un sur trois au Royaume-Uni, en Lettonie et en Finlande, et moins de un établissement sur dix en Belgique, en Italie et au Danemark.

\section{Le temps partiel : l'arrangement le plus répandu}

Le travail à temps partiel est l'arrangement de travail le plus connu en Europe. II a fait l'objet de nombreuses études, mais essentiellement du point de vue de son usage par les individus. Les études comparatives sur le temps partiel en Europe ont montré d'importantes différences dans le développement, I'usage, et la nature choisie ou subie du travail à temps partiel (O'Reilly et Fagan, 1998). Si les raisons liées aux politiques publiques et aux différences institutionnelles entre les pays ont été souvent étudiées, les pratiques des entreprises restent moins documentées ${ }^{*}$ ). Le travail à temps partiel est encouragé par la Stratégie européenne de I'emploi (définie au Sommet de Lisbonne en 1997), en tant que moyen pour articuler travail et famille, et avec I'objectif d'augmenter les taux d'emploi des femmes. Mais c'est également un moyen de répondre aux besoins organisationnels des entreprises en leur apportant un outil de flexibilité.

Un usage fréquent mais variable selon les pays ? L'exploitation de l'enquête ESWT fait apparaître que l'usage du travail à temps partiel est très répandu dans les entreprises en Europe : 64 \% des établissements de dix salariés et plus déclarent qu'au moins un de leurs salariés travaille à temps partiel, c'est-à-dire moins que la durée habituelle du travail à temps complet - $40 \%$ ont moins de $20 \%$ de leurs salariés à temps partiel et $24 \%$ plus de $20 \%$ de leurs salariés à temps partiel. L'usage

Graphique 4 - Nombre de problèmes associés à la présence de salariés en congés parentaux dans l'établissement (en \% d'établissements)

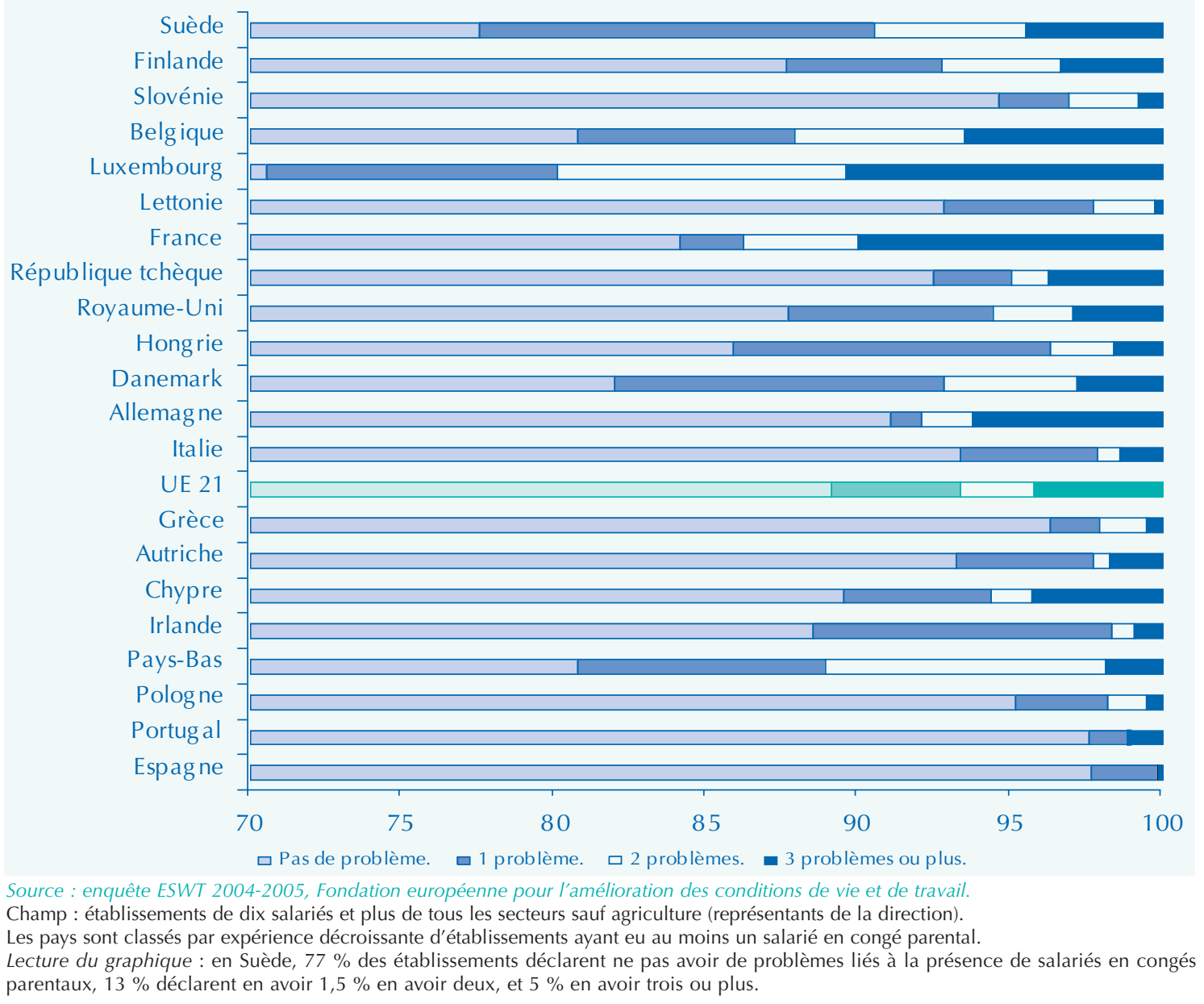

(*) Les études ayant porté sur les pratiques d'entreprises reposent essentiellement sur des enquêtes nationales, ou alors portent sur quelques pays uniquement (voir Allart et Bellemann, 2005, pour une comparaison de l'Allemagne et des Pays-Bas, ou Bosch et Lehndorff, 2005, qui retiennent quelques secteurs uniquement sur dix pays européens). 
de ce mode de travail varie selon les pays: les Pays-Bas ainsi que les pays scandinaves (Danemark et Suède) enregistrent les proportions les plus élevées d'établissements avec des salariés à temps partiel. À l'opposé, les établissements des pays d'Europe du Sud et de l'Est sont peu nombreux dans ce cas (graphique 5). Par ailleurs, les pays où les établissements recourent fortement au travail à temps partiel sont également ceux qui enregistrent des proportions de salariés à temps partiel supérieures à $20 \%$. Une analyse multivariée tenant compte de facteurs susceptibles d'influencer I'usage du temps partiel, comme le secteur d'activité, la taille de l'établissement ou la composition de la main-d'œuvre, indique que l'effet "pays » reste dominant dans I'explication de I'usage du temps partiel par les établissements. Les différences nationales dans les politiques publiques ou les relations industrielles, ou encore les différences culturelles, expliquent en grande partie I'usage de cette forme de travail.

\section{Le travail à temps partiel est plus répandu dans le secteur des services}

Au-delà de l'effet " pays ", on observe que l'usage du temps partiel est plus répandu dans les services (68\% des établissements) que dans l'industrie
(51\% des établissements). C'est dans les secteurs de la santé, des services sociaux, de l'éducation, des services à la personne, mais également dans I'hôtellerie et la restauration que l'usage du temps partiel est le plus répandu. Il augmente également avec la taille de l'établissement: alors que, dans près d'un établissement de petite taille sur deux (moins de cinquante salariés), les salariés travaillent à temps partiel, la proportion dépasse $80 \%$ pour les établissements de plus de deux cents salariés. La proportion de femmes parmi les salariés est également un facteur favorisant l'usage du temps partiel dans les établissements : dans $43 \%$ des établissements peu féminisés, les salariés sont à temps partiel alors que la proportion atteint $80 \%$ quand le taux de féminisation de la main-d'œuvre dépasse $60 \%$. Enfin, l'usage du travail à temps partiel est corrélé avec le recours à des formes flexibles de travail (contrats de travail courts, travail le week-end ou de nuit, travail en équipe), ainsi qu'avec des pratiques d'entreprises favorisant la conciliation (usage des congés parentaux, offre de services de garde).

\section{Besoins organisationnels de l'entreprise ou demandes des salariés}

Les établissements recourent au travail à temps

Graphique 5 - Proportion d'établissements ayant des salariés à temps partiel (en \% d'établissements)

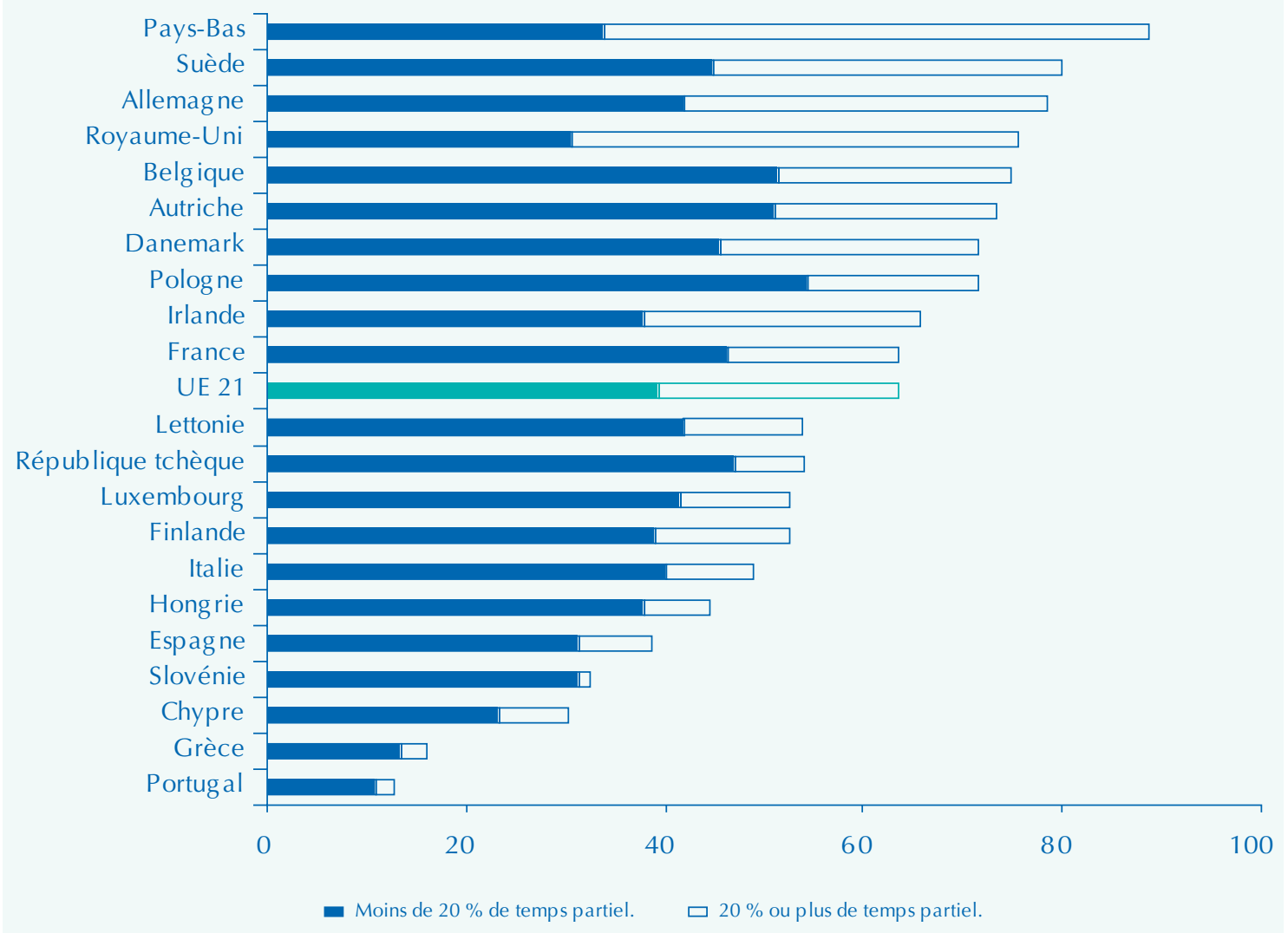

Source : enquête ESWT 2004-2005, Fondation européenne pour l'amélioration des conditions de vie et de travail.

Champ : établissements de dix salariés et plus de tous les secteurs sauf agriculture (représentants de la direction).

Lecture du graphique : aux Pays-Bas, $89 \%$ des établissements ont des salariés à temps partiel : $34 \%$ en ont moins de $20 \%$ et $55 \%$ en ont plus de $20 \%$. 
partiel pour des raisons qui relèvent soit des besoins organisationnels de l'entreprise, soit d'une demande des salariés (tableau 2). Selon les dirigeants, le temps partiel répond principalement aux besoins de l'établissement dans 34\% des établissements, alors que dans $39 \%$ des cas, il répond principalement aux souhaits des salariés ; $21 \%$ considèrent que les deux raisons sont d'égale importance. Lorsque le temps partiel vise à répondre aux besoins de l'établissement, il est plus souvent corrélé avec des horaires atypiques (travail de nuit ou le week-end), du travail en équipe et des possibilités limitées d'aménagements d'horaires. Il est le fait principalement d'établissements de plus petite taille, dans des secteurs tels que l'hôtellerie, la restauration, les services à la personne, l'éducation, la santé et les services sociaux, les transports. Les raisons invoquées par les employeurs pour justifier l'usage du temps partiel peuvent être approchées par l'identification des principaux groupes de personnes qui sont à temps partiel dans l'établissement: alors que I'on trouve plus souvent des mères d'enfants en âge préscolaire ou d'enfants plus grands à temps partiel dans les établissements utilisateurs, des proportions non négligeables d'établissements déclarent qu'ils ont des étudiants ou des jeunes à temps partiel, notamment dans les pays d'Europe du Sud, mais également au Royaume-Uni, en Irlande ou en Finlande. Bien que moins répandu, I'usage du temps partiel pour des personnes handicapées ou ayant des problèmes de santé est plus fréquent dans certains pays de l'Est que dans le reste de l'Europe. Enfin, bien que I'on trouve généralement moins d'hommes travaillant à temps partiel, le temps partiel masculin est plus répandu dans les pays d'Europe de l'Est, dans des établissements de grande taille du secteur des services, employant un grand nombre de salariés jeunes ou peu qualifiés.

L'organisation du travail à temps partiel est déterminante pour la qualité et la nature du travail. Le travail à temps partiel est plus couramment organisé sur la base d'horaires réduits quotidiennement, à part en Belgique, aux Pays-Bas et en Finlande, où il est plutôt organisé sous la forme de jours non travaillés. En revanche, une organisation flexible, selon les besoins de l'entreprise, est particulièrement répandue dans les établissements des pays d'Europe du Sud et de l'Est. Le mode d'organisation du travail à temps partiel reste sensible au contexte institutionnel (effet pays) bien que I'on retrouve une forte association entre organisation

Tableau 2 - Caractéristiques du travail à temps partiel

Raisons à l'introduction du temps partiel

Principalement les besoins organisationnels

Principalement la demande des salariés

Les deux d'égale importance

Principalement d'autres raisons

Ne sait pas ou sans réponse

Facile de passer de :

Temps partiel à temps complet

Temps complet à temps partiel (qualifiés)

Temps complet à temps partiel (peu qualifiés)

Organisation du temps partiel

Des heures fixes chaque jour

Autre organisation fixe (jours fixes)

Organisation flexible, fixée quelques jours avant selon

les besoins de l'entreprise

Autres formes

Groupes de personnes à temps partiel

Mères d'enfants scolarisés

Femmes sans enfant ou avec des enfants plus grands

Pères

Personnes s'occupant d'un proche âgé, malade ou handicapé

Personnes handicapées ou ayant des personnes de santé

Etudiants

Jeunes entrant sur le marché du travail

\begin{tabular}{|c|l|l|l|l}
$\begin{array}{c}\text { Moyenne } \\
\text { de I'UE 21 }\end{array}$ & $\begin{array}{c}\text { Pays avec la plus faible } \\
\text { proportion }\end{array}$ & \multicolumn{2}{|c|}{$\begin{array}{r}\text { Pays avec la plus forte } \\
\text { proportion }\end{array}$} \\
\hline 34 & PB (13) & Bel (15) & Grè (71) & Pol (66) \\
39 & Chy (13) & Grè (14) & Ita (63) & PB (62) \\
21 & Slov (7) & Pol (10) & Port (37) & Dan (30) \\
5 & Ire (1) & Dan (1) & Slov (37) & Tch (9) \\
1 & Grè (0) & Chy (0) & Lux (5) & Lett (5) \\
& & & & \\
27 & All (15) & Pol (16) & Tch (47) & Chy (45) \\
23 & Grè (6) & Port (7) & RU (39) & PB (36) \\
24 & Grè (8) & Port (9) & Suè (38) & RU (35) \\
& & & & \\
69 & Fin (37) & Lett (45) & Ita (85) & Slov (83) \\
38 & Slov (4) & Tch (11) & PB (74) & Bel (61) \\
& & & & \\
27 & Ita (7) & Slov (10) & Chy (40) & All (39) \\
7 & Grè (2) & Hon (2) & Fin (25) & Fra (13) \\
& & & & \\
64 & Port (12) & Slov (19) & Lux (81) & PB (78) \\
51 & Port (6) & Slov (11) & PB (71) & Suè (69) \\
21 & Slov (6) & Port (7) & PB (36) & Chy (33) \\
9 & Port (1) & Slov (1) & RU (18) & All (14) \\
17 & Port (0) & Grè (1) & Slov (56) & Tch (42) \\
18 & Slov (1) & Pol (5) & Irl (46) & Chy (41) \\
20 & Hon (5) & Ita (7) & RU (39) & Grè (34) \\
& & & &
\end{tabular}

Source : enquête ESWT 2004-2005, Fondation européenne pour l'amélioration des conditions de vie et de travail.

Champ : établissements de dix salariés et plus de tous les secteurs sauf agriculture (représentants de la direction et représentants des salariés). Lecture du tableau : en moyenne, dans les vingt et un pays de I'Union européenne (UE 21), $34 \%$ des employeurs déclarent qu'ils ont introduit le temps partiel dans leur établissement principalement pour des besoins organisationnels. Les pays où la proportion d'établissements donnant cette réponse est la plus faible sont les Pays-Bas et la Belgique, avec respectivement $13 \%$ et $15 \%$ d'employeurs. La proportion d'employeurs donnant cette réponse est la plus élevée en Grèce et en Pologne. 
flexible et autres arrangements flexibles d'organisation du travail (usage de contrats courts, travail de nuit, de week-end, en équipe, etc.). Enfin, I'usage du temps partiel en réponse aux besoins des salariés est largement nuancé par la très faible proportion d'établissements qui offrent des possibilités de réversibilité complète entre temps partiel et temps complet. En effet, seulement $9 \%$ des établissements des 21 pays de I'UE concernés par I'enquête déclarent que les salariés peuvent passer facilement, s'ils le demandent, de temps partiel à temps complet ou vice versa. Les pays où les possibilités de passage de l'un à l'autre sont les plus aisées sont la Suède, le Royaume-Uni, la France et I'Autriche (plus de $15 \%$ d'établissements). Ces possibilités sont plus fréquentes dans les établissements de grande taille où la main-d'œuvre est très féminisée, relevant du secteur des services, où I'usage du temps partiel est fréquent et où la maind'œuvre est jeune et qualifiée.

\section{Les offres de services aux salariés}

Parmi les prestations sous forme de services offerts aux salariés, il ne sera question ici que de celles qui touchent à l'accueil des enfants, sachant que cette offre est largement déterminée par l'existence de services publics. Seulement $4 \%$ d'établissements en moyenne offrent des services de garde d'enfants, ou d'autres formes d'aide à la garde (baby sitting). Cette offre est largement fonction de la taille de l'établissement (13\% d'établissements de 500 salariés et plus proposent de tels services) et du secteur d'activité (davantage dans les services, surtout dans la santé et le social, que dans I'industrie). Les Pays-Bas se détachent des autres pays avec $27 \%$ d'établissements offrant de tels services. Avec moins d'un établissement sur dix, on trouve ensuite le Royaume-Uni, I'Irlande et la Lettonie (graphique 6). En revanche, les pays du Sud de l'Europe, ainsi que la Suède et le Danemark ont des proportions très faibles d'établissements offrant de tels services.

\section{Une grande variabilité des pratiques des entreprises}

L'enquête ESWT portant sur les pratiques d'entreprises en matière de temps de travail et de condition de vie met en évidence la grande variabilité des pratiques des entreprises pour faciliter la conciliation travail-famille de leurs salariés. Cette variabilité tient en grande partie aux contextes institutionnels, aux formes différentes de régulation

Graphique 6 - Proportion d'établissements ayant des salariés à temps partiel (en \% d'établissements)

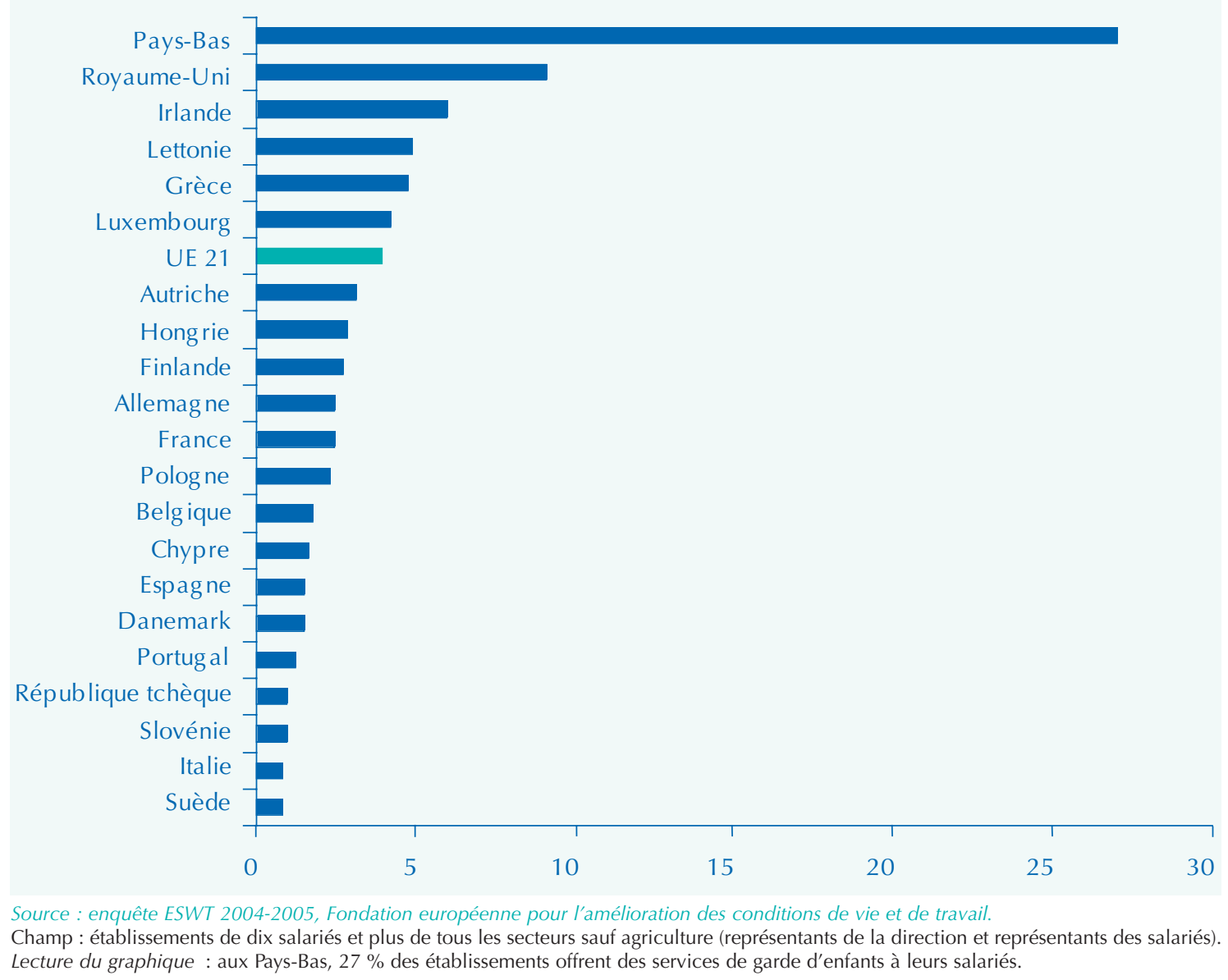


sociale et aux représentations collectives de la famille et de ses liens avec le travail. Les résultats montrent que les entreprises mettent en œuvre des aménagements d'horaires ou de temps de travail pour faciliter la conciliation entre travail et vie familiale de leurs salariés, tant sous forme d'horaires flexibles ou de travail à temps partiel. Cependant, il ressort également que ces pratiques répondent bien souvent aux besoins organisationnels des entreprises d'user de davantage de flexibilité, et que les entreprises ne se sont pas nécessairement dotées des moyens leur permettant d'intégrer la gestion de ces contraintes, comme par exemple pour les congés parentaux.

L'enquête portant sur les pratiques d'entreprises ne fournit pas d'informations sur les salariés concernés par les différentes mesures offertes, les éventuelles inégalités d'accès entre les salariés (les plus qualifiés et les autres par exemple), ni sur le ressenti des salariés vis-à-vis de ces mesures. II est donc très difficile d'évaluer l'importance respective des raisons avancées par les employeurs pour justifier I'introduction de ces mesures de conciliation qui restent marquées d'une certaine ambivalence. En matière de temps partiel ou d'horaires flexibles par exemple, il n'est pas possible de confronter les raisons de leur introduction avec ce qu'en disent les salariés. On peut juste souligner que les représentants du personnel répondent moins souvent que les employeurs qu'il s'agit d'une réponse aux besoins de conciliation des salariés. Ce manque plaide en faveur de la mise en place d'enquêtes appariées employeurs-salariés.

\section{Bibliographie}

Allart P. et Bellmann L., 2005, Employers' demand for part-time workers: incidence and motives in Germany and the Netherlands in Measuring the Information Society (sous la dir. de Ramioul M., Huws U. et Bollen A.), Leuven/Belgium:41-58.

Anxo D., Fagan C., Letablier M.-T., Perraudin C. et Smith M., 2007a, Parental leave in European companies. Establishment survey of working time 2004-2005, European Foundation for the Improvement of Living and Working Conditions, Luxembourg, Office for Official Publications of the European Communities.
Anxo D., Fagan C., Letablier M.T., Perraudin C. et Smith M., 2007b, Part-time work in European companies, Establishment survey of working time 2004-2005, European Foundation for the Improvement of Living and Working Conditions, Luxembourg, Office for Official Publications of the European Communities.

Bosch G. et Lehndorff S. (dir.), 2005, Working in the service sector - a tale of different worlds, London, Routledge.

Chung H., Kerkhofs M. et Ester P., 2007, Working time flexibility in European companies, Establishment survey of working time 2004-2005, European Foundation for the Improvement of Living and Working Conditions, Luxembourg, Office for Official Publications of the European Communities.

Fagan C., 2003, Working-time preferences and work-life balance in the EU: some policy considerations for enhancing the quality of life, Establishment survey of working time 2004-2005, European Foundation for the Improvement of Living and Working Conditions, Luxembourg Office for Official Publications of the European Communities.

Kümmerling A. et Lehndorff S., 2007, Extended and unusual working hours in European companies. Establishment survey of working time 2004-2005, European Foundation for the Improvement of Living and Working Conditions, Luxembourg Office for Official Publications of the European Communities.

Leber U. et Wagner A., 2007, Early and phased retirement in European companies. Establishment survey of working time 2004-2005, European Foundation for the Improvement of Living and Working Conditions, Luxembourg Office for Official Publications of the European Communities.

Lehndorff S., Kümmerling A. et Ramioul M., 2008, Social dialogue. Establishment survey of working time 2004-2005, European Foundation for the Improvement of Living and Working Conditions, Luxembourg Office for Official Publications of the European Communities (à paraître).

O'Reilly J. et Fagan C. (dir.), 1998, Part-time prospects: an international comparison of part-time work in Europe, North America and the Pacific Rim, London, Routledge.

Riedmann A., 2006, Working-time and work-life balance in European companies, Establishment survey of working time 2004-2005, European Foundation for the Improvement of Living and Working Conditions, Luxembourg Office for Official Publications of the European Communities. 